\title{
Pre-transplant MRD negativity predicts favorable outcomes of CAR-T therapy followed by haploidentical HSCT for relapsed/refractory acute lymphoblastic leukemia: a multi-center retrospective study
}

Houli Zhao ${ }^{1,2,3+}$, Jieping Wei ${ }^{1,2,3+}$, Guoqing Wei ${ }^{1,2,3+}, Y_{i}$ Luo $^{1,2,3}$, Jimin Shi ${ }^{1,2,3}$, Qu Cui ${ }^{4}$, Mingfeng Zhao ${ }^{5}$, Aibin Liang ${ }^{6}$, Qing Zhang ${ }^{7}$, Jianmin Yang ${ }^{8}$, Xin Li ${ }^{9}$, Jing Chen ${ }^{10}$, Xianmin Song ${ }^{11}$, Hongmei Jing ${ }^{12}$, Yuhua $\mathrm{Li}^{13}$, Siguo Hao ${ }^{14}$, Wenjun Wu $\mathbf{W}^{1,2,3}$, Yamin Tan ${ }^{1,2,3}$, Jian Yu ${ }^{1,2,3}$, Yanmin Zhao ${ }^{1,2,3}$, Xiaoyu Lai ${ }^{1,2,3}$, Elaine Tan Su Yin ${ }^{1,2,3}$, Yunxiong Wei ${ }^{5}$, Ping $\mathrm{Li}^{6}$, Jing Huang ${ }^{7}$, Tao Wang ${ }^{8}$, Didier Blaise ${ }^{15}$, Lei Xiao ${ }^{16}$, Alex H. Chang ${ }^{17}$, Arnon Nagler ${ }^{18}$, Mohamad Mohty ${ }^{19^{*}}$, He Huang ${ }^{1,2,3^{*}}$ and Yongxian $\mathrm{Hu}^{1,2,3^{*}}$

\begin{abstract}
Background: Consolidative allogeneic hematopoietic stem cell transplantation is a controversial option for patients with relapsed/refractory acute lymphoblastic leukemia after chimeric antigen receptor T cell (CAR-T) therapy. We performed a multicenter retrospective study to assess whether patients can benefit from haploidentical hematopoietic stem cell transplantation after CAR-T therapy.

Methods: A total of 122 patients after CAR-T therapy were enrolled, including 67 patients without subsequent transplantation (non-transplant group) and 55 patients with subsequent haploidentical hematopoietic stem cell transplantation (transplant group). Long-term outcome was assessed, as was its association with baseline patient characteristics.

Results: Compared with the non-transplant group, transplantation recipients had a higher 2-year overall survival (OS; 77.0\% versus 36.4\%; $P<0.001$ ) and leukemia-free survival (LFS; 65.6\% versus 32.8\%; $P<0.001$ ). Multivariate analysis showed that minimal residual disease (MRD) positivity at transplantation is an independent factor associated with poor LFS $(P=0.005)$, OS $(P=0.035)$, and high cumulative incidence rate of relapse $(P=0.045)$. Pretransplant MRD-negative recipients (MRD- group) had a lower cumulative incidence of relapse (17.3\%) than those (Continued on next page)
\end{abstract}

\footnotetext{
* Correspondence: mohamad.mohty@inserm.fr; huanghe@zju.edu.cn; 1313016@zju.edu.cn

${ }^{\dagger}$ Houli Zhao, Jieping Wei and Guoqing Wei contributed equally to this work.

${ }^{19}$ Sorbonne University, Saint-Antoine Hospital, INSERM UMRs 938, Paris,

France

'Bone Marrow Transplantation Center, The First Affiliated Hospital, School of Medicine, Zhejiang University, No.79 Qingchun Road, Hangzhou, China Full list of author information is available at the end of the article
}

C C The Author(s). 2020 Open Access This article is licensed under a Creative Commons Attribution 4.0 International License, which permits use, sharing, adaptation, distribution and reproduction in any medium or format, as long as you give appropriate credit to the original author(s) and the source, provide a link to the Creative Commons licence, and indicate if changes were made. The images or other third party material in this article are included in the article's Creative Commons licence, unless indicated otherwise in a credit line to the material. If material is not included in the article's Creative Commons licence and your intended use is not permitted by statutory regulation or exceeds the permitted use, you will need to obtain permission directly from the copyright holder. To view a copy of this licence, visit http://creativecommons.org/licenses/by/4.0/. The Creative Commons Public Domain Dedication waiver (http://creativecommons.org/publicdomain/zero/1.0/) applies to the data made available in this article, unless otherwise stated in a credit line to the data. 
(Continued from previous page)

in the non-transplant group (67.2\%; $P<0.001)$ and pre-transplant MRD-positive recipients (MRD+ group) $(65.8 \% ; P=$ 0.006). The cumulative incidence of relapse in MRD+ and non-transplant groups did not differ significantly $(P=$ 0.139). The 2-year LFS in the non-transplant, MRD+, and MRD- groups was 32.8\%, 27.6\%, and 76.1\%, respectively. The MRD - group had a higher LFS than the non-transplantation group $(P<0.001)$ and MRD+ group $(P=0.007)$, whereas the LFS in the MRD+ and non-transplant groups did not differ significantly $(P=0.305)$. The 2 -year OS of the MRD - group was higher than that of the non-transplant group (83.3\% versus 36.4\%; $P<0.001$ ) but did not differ from that of the MRD+ group (83.3\% versus 62.7\%; $P=0.069)$. The OS in the non-transplant and MRD+ groups did not differ significantly $(P=0.231)$.

Conclusion: Haploidentical hematopoietic stem cell transplantation with pre-transplant MRD negativity after CAR-T therapy could greatly improve LFS and OS in patients with relapsed/refractory acute lymphoblastic leukemia.

Trial registration: The study was registered in the Chinese clinical trial registry (ChiCTR1900023957).

Keywords: Chimeric antigen receptor T cell therapy, Haploidentical hematopoietic stem cell transplantation, Leukemia-free survival, Minimal residual disease negativity, Overall survival, Relapsed/refractory acute lymphoblastic leukemia

\section{Background}

Patients with relapsed/refractory acute lymphoblastic leukemia ( $R / R$ ALL) usually have a very poor prognosis with an expected median survival of less than 6 months, and the overall survival (OS) at 5 years is only $5-10 \%$ [1]. Complete remission (CR) rates after the first salvage chemotherapy are approximately $30-46 \%$, and these rates drop sharply to $18-25 \%$ after the second salvage chemotherapy. Allogeneic hematopoietic stem cell transplantation (allo-HSCT) is a potentially curative option for hematological malignancies and has improved the prognosis of R/R ALL over the past two decades. However, for patients who failed to achieve minimal residual disease (MRD) negativity before allo-HSCT, their 3-year OS and leukemia-free survival (LFS) were only $23.5 \%$ and $20.6 \%$, respectively [2]. Thus, novel therapeutic strategies to improve prognosis of these patients are urgently needed.

Recently, chimeric antigen receptor $\mathrm{T}$ cells (CAR-Ts) targeting CD19 or CD22 have been reported to successfully improve treatment outcomes for R/R ALL [3, 4]. In our previous clinical trial of CD19-targeted CAR-T therapy against R/R ALL, a CR rate of $92.3 \%$ was achieved [5]. However, data from long-term follow-ups in CAR-T trials show that relapse after CAR-T treatment still remains a predominant obstacle. Relapse rates of $20-70 \%$ were described when the follow-up period was sufficiently long [6]. The median LFS in patients with a low disease burden was 10.6 months, whereas that in patients with a high disease burden was 5.3 months [3]. With respect to the high relapse rates and the potentially unique relapse mechanisms after CAR-T treatment, consolidation therapy following CAR-T treatment may be considered as a necessary strategy, to reduce the risk of relapse and to maintain the status quo of CR.

Notably, consolidative allo-HSCT after CAR-T therapy is still a controversial option for improving long-term LFS.
Park et al. reported that of seventeen patients who underwent allo-HSCT after CAR-T therapy [3]. Relapse and transplantation-associated complications were the main causes of death for those who received CAR-T therapy before allo-HSCT, and the patients seemed not to benefit from allo-HSCT after CAR-T treatment [3]. Hay et al. found that allo-HSCT after CD19 CAR-T cell therapy was associated with a better LFS; however, they also reported a better LFS in MRD-negative CR patients who proceeded to allo-HSCT than those who did not [7]. In addition to alloHSCT from conventional donors, considerable progress has been made regarding haploidentical HSCT (haploHSCT) in recent years, and clinical outcomes of patients receiving haplo-HSCT have been reported to be similar to those receiving HLA-matched HSCT [8]. To date, the efficacy and safety profiles of haplo-HSCT after CAR-T treatment have not been assessed. Regarding the efficacy and transplant-associated complications, whether patients would benefit from allo-HSCT after CAR-T treatment remains controversial. And, no pre-transplant biomarker has been recommended to predict outcomes after transplantation. Further researches are needed to determine effectiveness of allo-HSCT consolidation therapy and to assess factors affecting long-term clinical outcomes.

Therefore, we designed a multicenter retrospective study to assess the efficacy and safety profiles of CAR-T therapy alone or CAR-T therapy followed by haploHSCT in patients with R/R ALL. We also determined potential prognostic factors associated with clinical outcomes in these patients.

\section{Methods}

Patients

This multicenter retrospective study included patients undergoing CAR-T treatment selectively followed by haplo-HSCT at 11 domestic centers in China from July 
2015 to December 1, 2019 (Supplementary Table 1; Fig. 1). Patient inclusion criteria were as follows: (1) age less than 70 years old; (2) relapsed or refractory CD19-positive ALL before CAR-T treatment; and (3) CD19 CAR-T treatment followed by achievement of MRD negativity in CR. Exclusion criteria included (1) pregnancy and lactation; (2) having conditions such as central nervous system diseases, clinically significant cardiovascular diseases, severe hepatic and renal dysfunctions, and various active infections; (3) having received systemic steroids in the previous 2 weeks (except for inhaled steroids) or gene therapies; (4) having any other conditions that might increase treatment risks; and (5) receiving CAR-T therapy followed by HLA-matched related HSCT or unrelated HSCT. The study was approved by the ethics review committee of each institution and retrospectively registered in the Chinese clinical trial registry (www.chictr.org. cn/showproj.aspx?proj=39004) (ChiCTR1900023957) on June 19, 2019. All participants provided written informed consent in accordance with the Declaration of Helsinki.

\section{Treatment protocol}

Anti-CD19 chimeric antigen receptor $\mathrm{T}$ cells constructed with 4-1BB costimulatory domain were generated via lentiviral vector from fresh leukapheresis material by Chinese biotech companies following the same manufacture standard as previously reported $[5,9]$. All the CAR-T cells required quality control according to the Code of manufacturing quality management for Chimeric Antigen Receptor $\mathrm{T}$ Cells (CAR-T cells) based-medicinal product formulated by China Medicinal Biotech Association [10] before discharge. After a lymphocyte-depleting chemotherapy with a fludarabinecyclophosphamide regimen, patients received an infusion of CD19 CAR-T cells. Response to therapy was assessed using morphological analysis and 6-color flow cytometry including CD10, CD19, CD20, CD34, CD38, and CD45. A sensitivity of $0.01 \%$ for MRD was achieved in all samples analyzed. Patients were then followed-up in outpatient departments for MRD detection (using flow cytometry) $1,2,3,6,12,18,24,36$, and 48 months after CAR-T therapy or haplo-HSCT. Depending on the willingness, economic background, and quality of life of patients, some patients received haplo-HSCT after CAR-T infusion. Patients who were going to receive the HSCT should provide MRD report within 1 week assessed by 6-color flow cytometry. Patients were divided into two groups: CAR-T treatment without transplant (non-transplant group) and CAR-T treatment followed by haploHSCT (transplant group).

Conditioning regimens included myeloablative regimens (busulfan and cyclophosphamide based and total body irradiation based) or nonmyeloablative regimens, as previously reported [11-14]. Most patients were younger, fit, and eligible for myeloablative conditioning. Patients eligible for myeloablative conditioning were typically older (depending on the center and time period, aged more than 60 years). After the conditioning

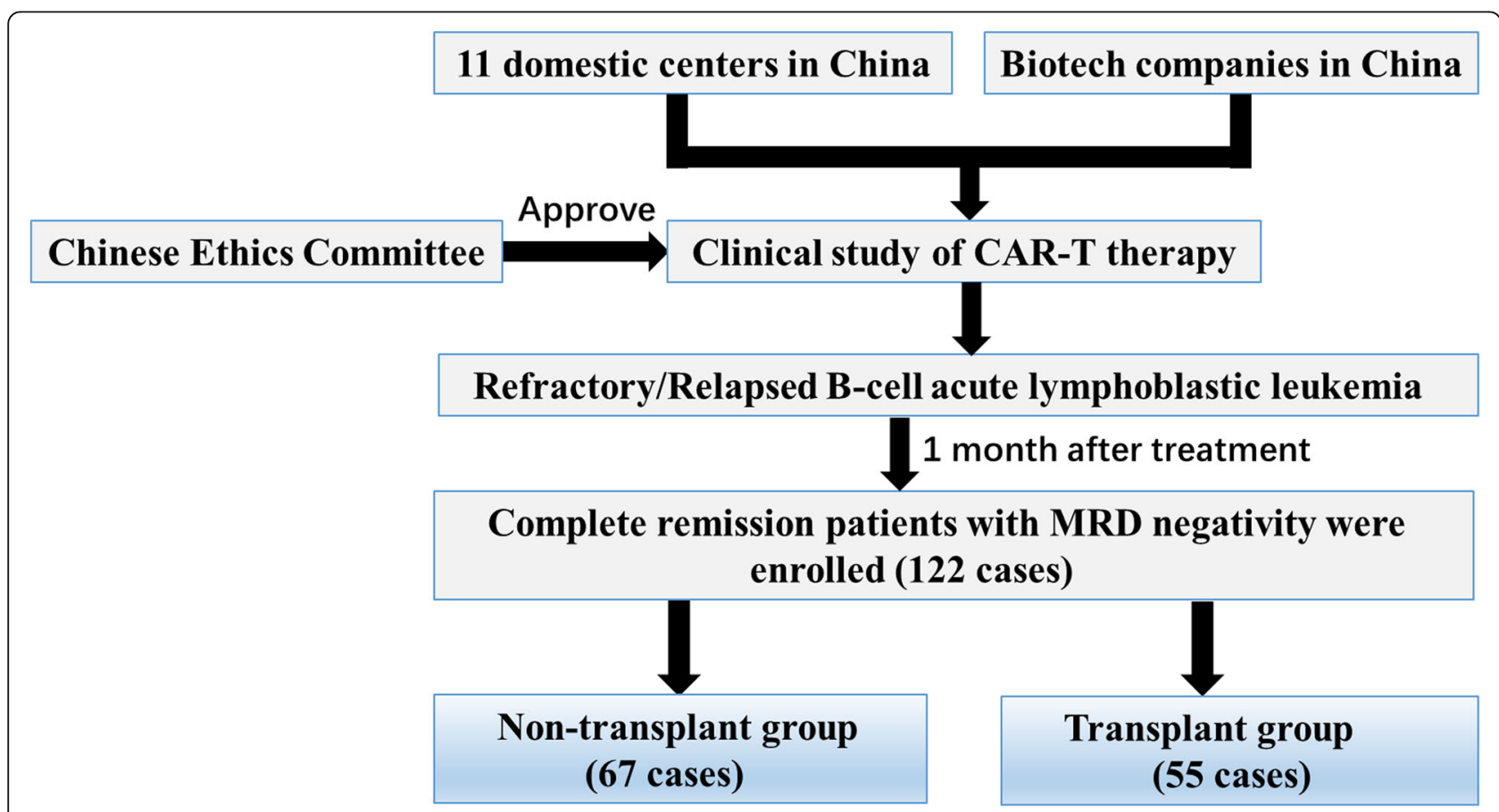

Fig. 1 Patient enrollment flow chart 
regimen, three patients received peripheral blood stem cells combined with bone marrow stem cells, and 54 patients received peripheral blood stem cells from haploidentical donors. Graft-versus-host-disease (GVHD) prophylactic regimens were determined by the individual transplant physician based upon disease-related and transplant-related considerations.

\section{Study end points, definitions, and statistical analysis}

The study aimed to assess the safety and efficacy of CAR- $T$ therapy alone or CAR-T therapy followed by haplo-HSCT for the treatment of R/R ALL. The primary end point was OS and LFS in patients with different pretransplant MRD status. The secondary endpoints included cumulative incidences of cytokine release syndrome (CRS), relapse, non-relapse mortality (NRM), acute GVHD (aGVHD), chronic GVHD (cGVHD), and viral infection after haplo-HSCT. For estimation of LFS, MRD-positive patients were not considered as having relapsed. MRD positivity was defined as having disease at a threshold of more than one ALL cell per 10,000 nuclear cells in the bone marrow, and LFS was defined as time to relapse or death, whichever occurs first. All measurement data were described using median and range and compared using $t$ tests. Enumeration data were presented as frequency (\%) and compared using chi-square tests. A competitive risk model was used to estimate the 100-day cumulative incidences of cGVHD requiring systemic steroid therapy and grade III-IV aGVHD and 2-year cumulative incidences of EpsteinBarr virus (EBV) or cytomegalovirus (CMV) infection and relapse. Follow-up time was estimated using the reverse Kaplan-Meier method, whereas OS and LFS were estimated using the Kaplan-Meier method. A Cox regression model was used to obtain the hazard ratio (HR) estimates and corresponding 95\% confidence intervals (CI) for OS and LFS. All $P$ values were two-sided, and results were considered statistically significant at $P<$ 0.05 . Data were analyzed using IBM SPSS Statistics 24 and $\mathrm{R}$ version 3.4.3.

\section{Results}

\section{Patient characteristics}

A total of 122 patients were enrolled in the study. The number of patients included in the non-transplant and transplant groups was 67 and 55, respectively (Fig. 1). Baseline characteristics are summarized in Table 1. The median age at the time of CAR-T therapy was 27 and 26 years in the non-transplant group and transplant group, respectively. Thirty-eight (31.1\%) patients were primary refractory to chemotherapy, and 26 (20.3\%) patients had previously undergone allo-HSCT. Twenty-five (20.5\%) patients were Philadelphia chromosome/BCR$A B L$ positive $(\mathrm{Ph}+)$ prior to CAR-T therapy. Prior to lymphodepletion, 87 (71.3\%) patients had morphological disease ( $\geq 5 \%$ blasts) in the bone marrow, with a median blast cell percentage of $33.8 \%$. There were only significant differences between the two groups in terms of prior allo-HSCT and source of CAR-T cells. The fraction of patients who had previously undergone alloHSCT was higher in the non-transplant group (22/67) than in the transplant group $(3 / 55)(P<0.001)$. Accordingly, the fraction of patients who received autologous CAR-T cells was higher in the transplant group (50/55) than in the non-transplant group (44/ 67) $(P=0.002)$. Multivariate analysis models for clinical outcome included these two variables and the variable "bridging to haplo-HSCT after CAR-T therapy." Neither prior allo-HSCT nor source of CAR-T cells were associated with clinical outcome, but bridging to haplo-HSCT after CAR-T therapy was associated with long LFS (HR: 0.244, 95\% CI: 0.136-0.437; $P<0.001$ ) and OS (HR: 0.275, 95\% CI: 0.142-0.531; $P$ $<0.001)$ as well as low cumulative incidence of relapse (HR: 0.163, 95\% CI: 0.081-0.329; $P<0.001$ ). In the transplant group, 15 patients $(27.3 \%)$ showed pretransplant MRD positivity. There were no statistically significant differences with respect to median age, gender, chromosomal aberrations, and gene mutations between the two groups $(P>0.05$; Table 1$)$.

\section{CAR-T therapy-associated toxicities}

The most common CAR-T therapy-associated toxicity was CRS. The peak of CRS occurred in a median time of 6 days after CAR-T infusion. Complicated CRS of grades $0,1-3$, and $4-5$ was observed in 9,108 , and 5 patients, respectively. Platelet count below $10^{4} / \mu \mathrm{L}$ with a median onset time of 2 days after CAR-T therapy occurred in 77 (63.1\%) patients, and the condition lasted for a median time of 8 days. Neutropenia occurred at a median time of 2 days after CAR-T infusion in 91 (74.6\%) patients and lasted for a median time of 5 days after onset (Supplementary Table 2). Except for the 9 patients with grade 0 CRS, patients $(n=113)$ suffered from pyrexia and were monitored by examination of bacterial and fungal cultures. Three patients had septicemia due to Burkholderia cepacia, Trichosporon asahii, and Klebsiella pneumoniae, whereas three patients suffered from pneumonia, with Stenotrophomonas maltophilia, alpha streptococcus/Neisseria pharyngis, and Klebsiella pneumoniae infections in the sputum. Salmonella bovismorbificans and Candida albicans infections in the stools were recorded in one patient with diarrhea. There were no statistically significant differences with respect to factors associated with CAR-T therapy between the two groups (Supplementary Table 2). 
Table 1 Patient characteristics between non-transplant group and transplant group

\begin{tabular}{|c|c|c|c|}
\hline & & & $P$ \\
\hline Characteristics & Non-transplant $(n=67)$ & Transplant $(n=55)$ & Non-transplant vs transplant \\
\hline Gender, $n(\%)$ & & & 0.153 \\
\hline Male & $34(50.7)$ & $35(63.6)$ & \\
\hline Female & $33(49.3)$ & $20(36.4)$ & \\
\hline Age, years & & & 0.332 \\
\hline Median (range) & $27(9,65)$ & $26(3,65)$ & \\
\hline Hyperploidy, n (\%) & $3(4.5)$ & $3(5.5)$ & 0.804 \\
\hline Hypoplodiy, n (\%) & $1(1.5)$ & $1(1.8)$ & 0.888 \\
\hline Complex karyotype, $n$ (\%) & $4(6.0)$ & $2(3.6)$ & 0.553 \\
\hline iAMP $21^{a}, n(\%)$ & $1(1.5)$ & $1(1.8)$ & 0.888 \\
\hline ETV6-RUNX1, n (\%) & $1(1.5)$ & $0(0)$ & 0.363 \\
\hline $\mathrm{E} 2 \mathrm{~A}-\mathrm{PBX} 1, n(\%)$ & $1(1.5)$ & $0(0)$ & 0.363 \\
\hline MYC mutation, $n(\%)$ & $1(1.5)$ & $0(0)$ & 0.363 \\
\hline HOX11 mutation, $n(\%)$ & $1(1.5)$ & $0(0)$ & 0.363 \\
\hline KMT2A rearranged, $n(\%)$ & $3(4.5)$ & $5(9.1)$ & 0.306 \\
\hline BCR-ABL1, n (\%) & $11(16.4)$ & $11(20.0)$ & 0.609 \\
\hline Ph like, $n(\%)$ & $1(1.5)$ & $4(7.3)$ & 0.109 \\
\hline IgH rearranged, $n(\%)$ & $1(1.5)$ & $0(0)$ & 0.363 \\
\hline IKZF1 mutation, n\% & $3(4.5)$ & $7(12.7)$ & 0.098 \\
\hline Poor-risk cytogenetics ${ }^{\mathrm{b}}, n(\%)$ & $18(26.9)$ & $21(38.2)$ & 0.182 \\
\hline Good-risk cytogenetics ${ }^{c}, n(\%)$ & $2(3.0)$ & $2(3.6)$ & 0.841 \\
\hline Primary refractory to chemotherapy, $n$ (\%) & $17(25.4)$ & $21(38.2)$ & 0.128 \\
\hline Total number of relapses before CAR-T & & & 0.405 \\
\hline Median (range) & $1(0,6)$ & $1(0,3)$ & \\
\hline Prior lines of therapy & & & 0.888 \\
\hline Median (range) & $4(2,15)$ & $3(2,9)$ & \\
\hline Prior allo-HSCT, $n(\%)$ & $22(32.8)$ & $3(5.5)$ & $<0.001$ \\
\hline Extramedullary infiltration, $n(\%)$ & $9(13.4)$ & $3(5.5)$ & 0.141 \\
\hline Platelet count before CAR-T, $10^{9} / \mathrm{L}$ & & & 0.339 \\
\hline Median (range) & $130(15,412)$ & $140(12,389)$ & \\
\hline PLT $<1 *$ LLN before CAR-T, $n(\%)(n=66+52)$ & $28(42.4)$ & $15(28.8)$ & 0.128 \\
\hline LDH > 1*ULN before CAR-T, $n(\%)(n=66+52)$ & $26(39.4)$ & $25(48.1)$ & 0.345 \\
\hline Blast cells in bone marrow before CAR-T & & & 0.766 \\
\hline Median (range) & $22.5 \%(0,97.0 \%)$ & $16.0 \%(0,90.0 \%)$ & \\
\hline CAR-T cell dose, $10^{6} / \mathrm{kg}$ & & & 0.853 \\
\hline Median (range) & $3.0(0.3,25.0)$ & $5.0(0.1,30.4)$ & \\
\hline Source of CAR-T cells, $n(\%)$ & & & 0.002 \\
\hline Autologous & $44(65.7)$ & $50(90.9)$ & \\
\hline Recipient-derived allogenic & $15(22.4)$ & $3(5.5)$ & \\
\hline Donor-derived allogenic & $7(10.4)$ & $0(0)$ & \\
\hline Third-party & $1(1.5)$ & $2(3.6)$ & \\
\hline
\end{tabular}

${ }^{\mathrm{a}}$ iAMP21 intrachromosomal amplification of chromosome 21

${ }^{b}$ Poor-risk cytogenetics are defined as hypodiploidy, KMT2A rearranged, $t(v ; 14 q 23) / l g H$ rearranged, $t(9 ; 22)(q 34 ; q 11.2)$ : BCR-ABL1, complex karyotype, Ph-like, and iAMP21

'Good-risk cytogenetics are defined as hyperdiploidy, and t(12;21)(p13;q22): ETV6-RUNX1 


\section{Long-term clinical outcomes}

Up to the last follow-up, in the non-transplant group, 44 (65.7\%) patients relapsed, whereas 11 (20.0\%) patients relapsed with CD19 negativity. The 2-year cumulative incidence of relapse in the non-transplant group and transplant group was $67.2 \%$ and $27.6 \%(P<0.001)$, respectively (Fig. 2a). The 2-year cumulative incidence of NRM in the non-transplant group and transplant group was 0 and $6.8 \%(P=0.047)$, respectively (Fig. $2 b)$. The $2-$ year LFS and OS of patients were higher in the transplant group than in the non-transplant group $(65.6 \%$ versus $32.8 \%$ and $77.0 \%$ versus $36.4 \%$, respectively; $P<$ 0.001; Fig. 2c and d).

\section{Factors associated with clinical outcomes}

Hazard ratios of prognostic factors associated with LFS, OS, and cumulative incidence of relapse obtained using univariate and multivariate analysis are shown in Supplementary Tables 3-5 and Table 2 . MRD positivity at the time of haplo-HSCT (HR: 4.466, 95\% CI: 1.561-12.776;
$P=0.005)$ and age beyond or equal to 40 years before CAR-T therapy (HR: 4.706, 95\% CI: 1.6301-13.586; $P=$ 0.004 ) are the two independent prognostic factors associated with poor LFS. MRD positivity at the time of haploHSCT (HR: 3.699, 95\% CI: 1.095-12.498; $P=0.035$ ) and age beyond or equal to 40 years (HR: $7.110,95 \% \mathrm{CI}$ : 2.178-23.210; $P=0.001$ ) were two independent prognostic factors associated with poor OS. MRD positivity at the time of haplo-HSCT (HR: 4.190, 95\% CI: 1.032-17.013; $P$ $=0.045)$ and more than one relapse before CAR-T infusion (HR: 4.450, 95\% CI: 1.069-18.527; $P=0.040$ ) were two independent prognostic factors associated with higher cumulative incidence of relapse. For NRM, there were only 5 cases after transplantation which was not adequate for statistical analysis. Complex karyotype, high risk by genetics, primary refraction to chemotherapy, prior lines of chemotherapy, prior allo-HSCT, donor age, and blast cells in the bone marrow before CAR-T infusion were not significant prognostic factors for LFS, OS, or cumulative incidence of relapse.
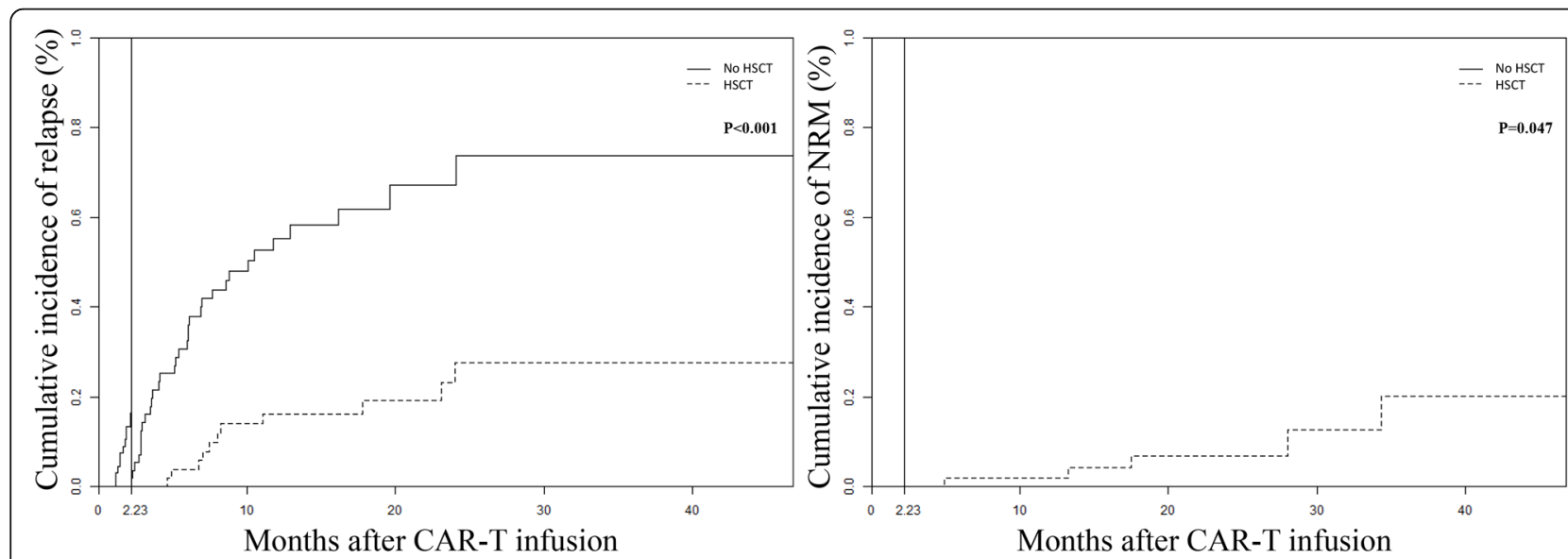

A

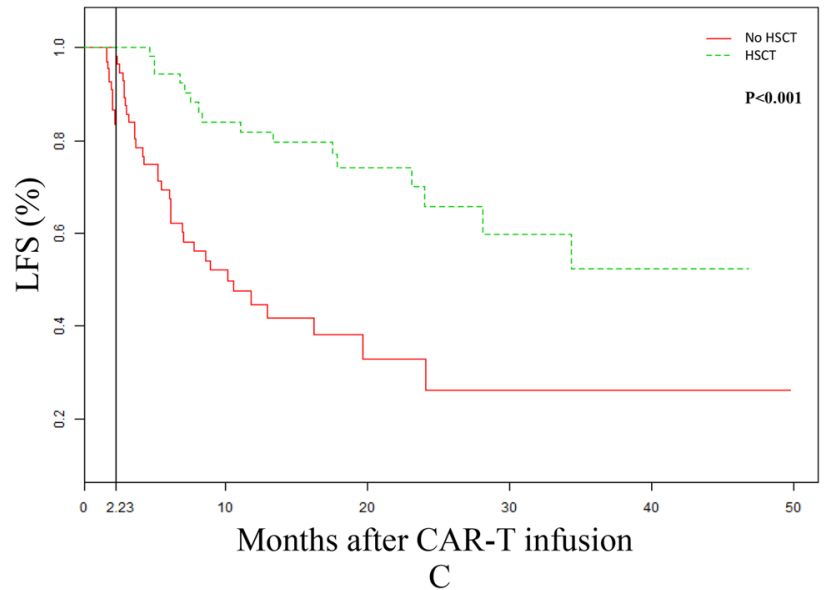

$\mathrm{B}$

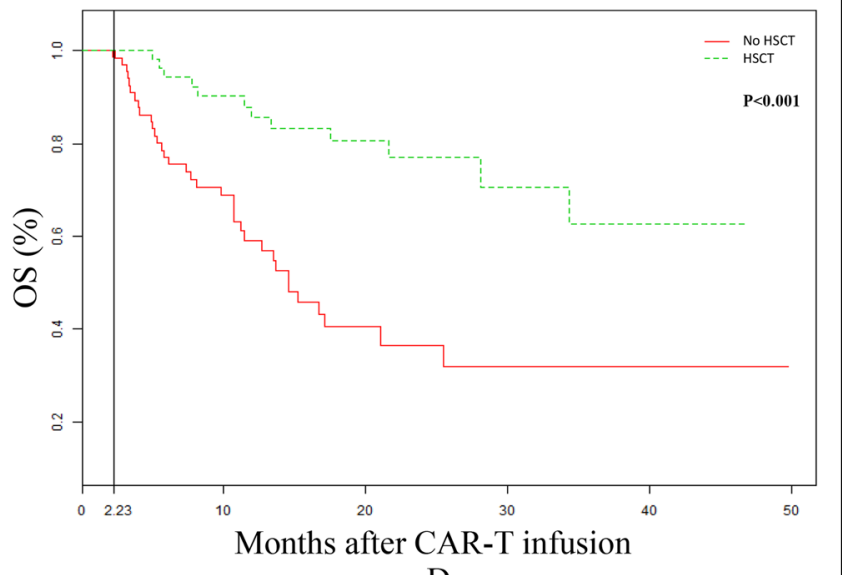

$\mathrm{D}$

Fig. 2 Landmark analysis, cumulative incidence of relapse, non-relapse mortality, leukemia-free survival (LFS) and overall survival (OS) between the non-transplant group and the transplant group. The cut-off value was set at the median time from CAR-T cell infusion to haplo-HSCT (2.23 months). No HSCT: non-transplant group, HSCT: transplant group 
Table 2 Multivariable analyses for factors impacting LFS, OS, and cumulative incidence of relapse in patients who received CAR-T therapy followed by haplo-HSCT

\begin{tabular}{llll}
\hline Factors & & Hazard ratio $(95 \% \mathrm{Cl})$ & $P$ value \\
\hline LFS & & & \\
Age & $\geq 40$ vs. $<40$ & $4.706(1.630-13.586)$ & $\mathbf{0 . 0 0 4}$ \\
MRD before HSCT & Positive vs. negative & $4.466(1.561-12.776)$ & \\
OS & & & $\mathbf{0 . 0 0 5}$ \\
Age & $\geq 40$ vs. $<40$ & $7.110(2.178-23.210)$ & $\mathbf{0 . 0 0 1}$ \\
MRD before HSCT & Positive vs. negative & $3.699(1.095-12.498)$ & $\mathbf{0 . 0 3 5}$ \\
Cumulative incidence of relapse & & & \\
$\quad$ Total number of relapses before CAR-T & $>1$ vs. $\leq 1$ & $4.450(1.069-18.527)$ & $\mathbf{0 . 0 4 0}$ \\
MRD before HSCT & Positive vs. negative & $4.190(1.032-17.013)$ & $\mathbf{0 . 0 4 5}$ \\
\hline
\end{tabular}

\section{Minimal residual disease negativity and favorable outcomes}

Patients with pre-transplant MRD negativity and MRD positivity were divided into MRD- group and $\mathrm{MRD}+$ group, respectively. The 2-year cumulative incidence of relapse was $67.2 \%, 65.8 \%$, and $17.3 \%$ in the non-transplant group, MRD+ group, and MRDgroup, respectively. Patients in the MRD- group had a lower cumulative incidence of relapse than those in the non-transplant group and in MRD+ group $(P<$ $0.001)$; the cumulative incidence of relapse in patients in the MRD+ group and non-transplant group did not differ significantly $(P=0.139$; Fig. 3a). The 2-year cumulative incidence of NRM in the MRD- group did not differ from that in the non-transplant group or MRD+ group (6.6\% versus 0 and $6.6 \%$ versus $6.7 \%$, respectively; $P=0.052$ and 0.807 , respectively; Fig. $3 \mathrm{~b})$.

The 2-year LFS in the non-transplant group, MRD+ group, and MRD- group was $32.8 \%, 27.6 \%$, and $76.1 \%$, respectively (Table 3 ). Patients in the MRD- group had a higher LFS than those in the non-transplant group $(P$ $<0.001)$ and MRD+ group $(P=0.007)$; LFS in the $\mathrm{MRD}+$ group and non-transplant group did not differ significantly $(P=0.305)$ (Fig. 3c). The 2 -year OS in the MRD-group was higher than that in the non-transplant group (83.3\% versus $36.4 \% ; P<0.001)$ but did not differ from that in the MRD+ group (83.39\% versus $62.7 \%$; $P=$ $0.069)$; the 2-year OS in the non-transplant group and MRD+ group did not differ significantly $(P=0.231$; Fig. 3d; Table 3).

\section{Engraftment and chimerism after haplo-HSCT}

Fifty-five patients who received haplo-HSCT at a median time of 67 days after CAR-T cell infusion (range 34-345 days) were included. The median follow-up time was 613 days after CAR-T therapy (range 1001403 days). Baseline characteristics at the time of haplo-HSCT are summarized in Table 4. Patients in the MRD+ and MRD- groups were well matched, and there were no significant differences between the two cohorts in terms of underlying disease, time from CAR-T infusion to haplo-HSCT, type of graft, conditioning regimen, GVHD prophylactic regimen, or CD34+ cell dose. After myeloid recovery, all patients achieved sustained, full donor chimerism by day +30 after haplo-HSCT. The median time to neutrophils $\geq$ $0.5 \triangleright 10^{9} / \mathrm{L}$ after transplantation was 13 days (10-21 days), and the median time to platelets $\geq 20 \diamond 10^{9} / \mathrm{L}$ after transplantation was 16 days (9-65 days). Two patients suffered from primary platelet dysfunction.

Table 3 Results of main clinical outcomes after CAR-T

\begin{tabular}{|c|c|c|c|c|c|c|}
\hline Group & $\begin{array}{l}\text { 1-year cumulative incidence } \\
\text { of relapse (\%) }\end{array}$ & $\begin{array}{l}\text { 2-year cumulative incidence } \\
\text { of relapse (\%) }\end{array}$ & $\begin{array}{l}\text { 1-year LFS } \\
(\%)\end{array}$ & $\begin{array}{l}\text { 2-year LFS } \\
(\%)\end{array}$ & $\begin{array}{l}\text { 1-year OS } \\
(\%)\end{array}$ & $\begin{array}{l}\text { 2-year OS } \\
(\%)\end{array}$ \\
\hline Non-transplant & 55.3 & 67.2 & 44.7 & 32.8 & 59.1 & 36.4 \\
\hline MRD+ & 38.2 & 65.8 & 55.2 & 27.6 & 62.7 & 62.7 \\
\hline MRD- & 8.4 & 17.3 & 91.6 & 76.1 & 94.3 & 83.3 \\
\hline$P$ between non-transplant and MRD+ & & 0.139 & & 0.305 & & 0.231 \\
\hline$P$ between non-transplant and MRD- & & $<0.001$ & & $<0.001$ & & $<0.001$ \\
\hline$P$ between $M R D+$ and MRD- & & 0.006 & & 0.007 & & 0.069 \\
\hline
\end{tabular}



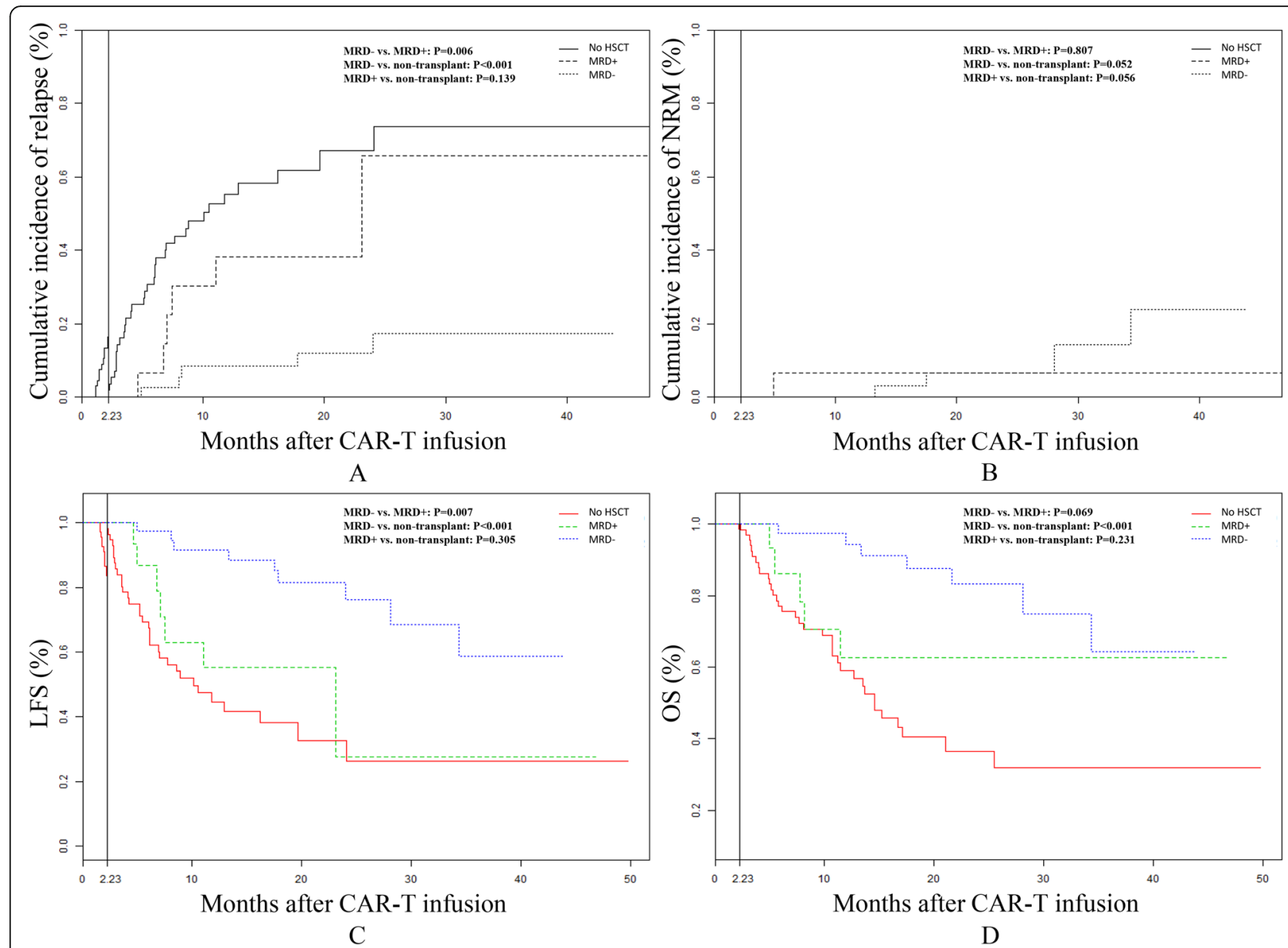

Fig. 3 Landmark analysis, cumulative incidence of relapse, non-relapse mortality, leukemia-free survival (LFS) and overall survival (OS) among the non-transplant group, MRD+ group and MRD- group. The cut-off value was set at the median time from CAR-T cell infusion to haplo-HSCT (2.23 months). No HSCT: non-transplant group, MRD+: MRD+ group, MRD-: MRD- group

\section{Acute and chronic graft-versus-host-disease}

Supplementary Table 6 summarizes the complications after transplantation. The 100-day cumulative incidence of grade III-IV aGVHD was 7.3\% (Supplementary Figure 1A). The 2-year cumulative incidence of cGVHD requiring systemic steroid therapy was $25.5 \%$ (Supplementary Figure 1B).

\section{Infection complications after transplantation}

The percentages of patients experiencing at least one bacterial and one invasive fungal infection were 14.0\% (8/57) and $5.3 \%(3 / 57)$, respectively. Bacterial infections with Escherichia coli $(n=2)$, Enterococcus faecium $(n=2)$, Pseudomonas aeruginosa $(n=2)$, Gemella haemolysans ( $n$ $=1)$, Stenotrophomonas maltophilia $(n=1)$, Nocardia farcinica $(n=1)$, and Staphylococcus haemolyticus $(n=1)$ were recorded. All patients responded to antibiotics. Viral infection was also recorded in this population. The 2-year cumulative incidence of CMV and EBV viremia was 56.1\% and 57.5\%, respectively (Supplementary Figure 1C and D, Supplementary Table 6).

\section{Other complications after transplantation}

Seven patients suffered from cystitis. Two patients developed EBV-associated post-transplant lymphoproliferative disorders and received rituximab with rapid response. One patient developed seizures during the conditioning period. One patient developed immune thrombocytopenia 4 months after transplantation but was refractory to steroids. This patient then received intravenous high-dose immunoglobulin therapy and had a rapid remission.

\section{Discussion}

At present, patients with R/R ALL have a low likelihood of being cured with salvage regimens or available investigational agents. CAR-T therapy has emerged as a rescue for induction therapy in patients with R/R ALL, and clinical studies have reported favorable outcomes in 
Table 4 Patient characteristics among 3 groups

\begin{tabular}{|c|c|c|c|c|c|c|}
\hline \multirow[b]{2}{*}{ Characteristics } & \multirow[b]{2}{*}{$\begin{array}{l}\text { Non-transplant } \\
(n=67)\end{array}$} & \multirow[b]{2}{*}{$\begin{array}{l}\text { MRD+ } \\
(n=15)\end{array}$} & \multirow[b]{2}{*}{$\begin{array}{l}\text { MRD- } \\
(n=40)\end{array}$} & \multicolumn{3}{|l|}{ P } \\
\hline & & & & $\begin{array}{l}\text { Non-transplant } \\
\text { vs MRD+ }\end{array}$ & $\begin{array}{l}\text { Non-transplant } \\
\text { vs MRD- }\end{array}$ & $\begin{array}{l}\text { MRD- vs } \\
\text { MRD+ }\end{array}$ \\
\hline Gender, $n(\%)$ & & & & 0.112 & 0.353 & 0.360 \\
\hline Male & $34(50.7)$ & $11(73.3)$ & $24(60.0)$ & & & \\
\hline Female & $33(49.3)$ & $4(26.7)$ & $16(40.0)$ & & & \\
\hline Age, years & & & & 0.384 & 0.248 & 0.931 \\
\hline Median (range) & $27.0(9.0,65.0)$ & $26.0(7.0,65.0)$ & $26.5(3.0,63.0)$ & & & \\
\hline Hyperploidy, n (\%) & $3(4.5)$ & $1(6.7)$ & $2(5.0)$ & 0.722 & 0.901 & 0.808 \\
\hline Hypoplodiy, n (\%) & $1(1.5)$ & $0(0)$ & $1(2.5)$ & 0.634 & 0.710 & 0.537 \\
\hline Complex karyotype, $n(\%)$ & $4(6.0)$ & $0(0)$ & $2(5.0)$ & 0.332 & 0.833 & 0.378 \\
\hline iAMP $21^{\mathrm{a}}, n(\%)$ & $1(1.5)$ & $1(6.7)$ & $0(0)$ & 0.240 & 0.438 & 0.099 \\
\hline ETV6-RUNX1, n (\%) & $1(1.5)$ & $0(0)$ & $0(0)$ & 0.634 & 0.438 & NA \\
\hline E2A-PBX1, n (\%) & $1(1.5)$ & $0(0)$ & $0(0)$ & 0.634 & 0.438 & NA \\
\hline MYC mutation, $n(\%)$ & $1(1.5)$ & $0(0)$ & $0(0)$ & 0.634 & 0.438 & NA \\
\hline HOX11 mutation, $n(\%)$ & $1(1.5)$ & $0(0)$ & $0(0)$ & 0.634 & 0.438 & NA \\
\hline KMT2A rearranged, $n(\%)$ & $3(4.5)$ & $1(6.7)$ & $4(10.0)$ & 0.722 & 0.264 & 0.702 \\
\hline BCR-ABL1, n (\%) & $11(16.4)$ & $3(20.0)$ & $8(20.0)$ & 0.739 & 0.639 & 1.000 \\
\hline Ph like, $n(\%)$ & $1(1.5)$ & $1(6.7)$ & $3(7.5)$ & 0.240 & 0.113 & 0.916 \\
\hline IgH rearranged, $n(\%)$ & $1(1.5)$ & $0(0)$ & $0(0)$ & 0.634 & 0.438 & NA \\
\hline IKZF1 mutation, n\% & $3(4.5)$ & $3(20.0)$ & $4(10.0)$ & 0.037 & 0.264 & 0.322 \\
\hline Poor-risk cytogenetics ${ }^{\mathrm{b}}, n(\%)$ & $18(26.9)$ & $6(40.0)$ & $15(37.5)$ & 0.312 & 0.249 & 0.865 \\
\hline Good-risk cytogenetics', $n(\%)$ & $2(3.0)$ & $0(0)$ & $1(2.5)$ & 0.492 & 0.883 & 0.462 \\
\hline Primary refractory to chemotherapy, $n$ (\%) & $17(25.4)$ & $7(46.7)$ & $14(35.0)$ & 0.101 & 0.288 & 0.428 \\
\hline Total number of relapses before CAR-T & & & & 0.148 & 0.120 & 0.633 \\
\hline Median (range) & $1(0,6)$ & $1(0,2)$ & $1(0,3)$ & & & \\
\hline Prior lines of therapy & & & & 0.519 & 0.491 & 0.250 \\
\hline Median (range) & $4(2,15)$ & $4(2,9)$ & $3(2,9)$ & & & \\
\hline Prior allo-HSCT, $n(\%)$ & $22(32.8)$ & $2(13.3)$ & $1(2.5)$ & 0.133 & $<0.001$ & 0.115 \\
\hline Extramedullary infiltration, $n$ (\%) & $9(13.4)$ & $2(13.3)$ & $1(2.5)$ & 0.992 & 0.060 & 0.115 \\
\hline $\begin{array}{l}\text { Platelet count before CAR-T, } 10^{9} / \mathrm{L} \\
(N=66+14+38)\end{array}$ & & & & 0.511 & 0.186 & 0.799 \\
\hline Median (range) & $130(15,412)$ & $139(12,302)$ & $140(21,389)$ & & & \\
\hline $\begin{array}{l}\text { PLT }<1 * \text { LLN before CAR-T, } n(\%) \\
(N=66+14+38)\end{array}$ & $28(42.4)$ & $4(26.7)$ & $11(27.5)$ & 0.337 & 0.172 & 0.979 \\
\hline $\begin{array}{l}\text { LDH }>1 * \text { ULN before CAR-T, } n(\%) \\
(n=66+14+38)\end{array}$ & $26(39.4)$ & $6(40.0)$ & $19(47.5)$ & 0.810 & 0.293 & 0.647 \\
\hline Blast cells in bone marrow before CAR-T & & & & 0.802 & 0.867 & 0.731 \\
\hline Median (range) & $22.5 \%(0,97.0 \%)$ & $6.6 \%(0.1 \%, 89.4 \%)$ & $22.3 \%(0,90.0 \%)$ & & & \\
\hline CAR-T cell dose, $10^{6} / \mathrm{kg}$ & & & & 0.340 & 0.302 & 0.881 \\
\hline Median (range) & $3.0(0.3,25.0)$ & $5.4(1.0,11.1)$ & $5.0(0.1,30.4)$ & & & \\
\hline Source of CAR-T cells, $n(\%)$ & & & & 0.438 & 0.046 & 0.208 \\
\hline Autologous & $51(76.1)$ & $12(80.0)$ & $38(95.0)$ & & & \\
\hline Recipient-derived allogenic & $9(13.4)$ & $2(13.3)$ & $1(2.5)$ & & & \\
\hline Donor-derived allogenic & $6(9.0)$ & $0(0)$ & $0(0)$ & & & \\
\hline Third-party & $1(1.5)$ & $1(6.7)$ & $1(2.5)$ & & & \\
\hline
\end{tabular}

aAMP21 intrachromosomal amplification of chromosome 21

bPoor-risk cytogenetics are defined as hypodiploidy, KMT2A rearranged, $t(v ; 14 q 23) / l g H$ rearranged, $t(9 ; 22)(q 34 ; q 11.2)$ : BCR-ABL1, complex karyotype, Ph-like, and iAMP21

${ }^{c}$ Good-risk cytogenetics are defined as hyperdiploidy, and t(12;21)(p13;q22): ETV6-RUNX1 
those cases [15-17]. However, relapse after CAR-T treatment remarkably decreases the long-term LFS and OS. As reported, allo-HSCT after CAR-T therapy has the potential to reduce the risk of relapse and improve long-term LFS and OS. Nevertheless, up to date, limited data are available on the clinical outcomes of this combination strategy. In this study, for the first time to our knowledge, we retrospectively reviewed data from CAR$\mathrm{T}$ therapy alone or CAR-T therapy followed by haploHSCT for R/R B-ALL in 11 domestic centers in China. Our results showed that patients could greatly benefit from haplo-HSCT after CAR-T therapy. Further analysis indicated that the OS and LFS of patients who received CAR-T treatment followed by haplo-HSCT and achieved pre-transplant MRD negativity tend to be higher than those treated with CAR-T therapy without allo-HSCT, or those who received CAR-T treatment followed by haplo-HSCT and showed pre-transplant MRD positivity. For patients receiving CAR-T treatment followed by haplo-HSCT, MRD negativity at the time of haploHSCT was a significant prognostic factor associated with higher LFS and OS. In addition to the beneficial longterm outcomes, risks of treatment-related toxicities were not increased.

Patients with R/R ALL who achieved MRD negativity after chemotherapy and subsequently underwent alloHSCT are reported to have the best clinical outcomes $[18,19]$. Pavlů et al. reported that compared with pretransplant MRD negativity, pre-transplant MRD positivity was associated with significantly lower OS $(61 \%$ versus $67 \%$ ) and LFS (50\% versus $58 \%$ ), and with a higher cumulative incidence of relapse (32\% versus $24 \%$ ) at 2 years post transplantation [20]. In this study, all patients were in the second or third complete remission (CR2 or CR3) or were primary refractory; the 2-year OS, LFS, and cumulative incidence of relapse for patients with pre-transplant MRD negativity and MRD positivity were $83.3 \%$ versus $62.7 \%, 76.1 \%$ versus $27.6 \%$, and $17.3 \%$ versus $65.8 \%$, respectively. The significantly high OS, LFS, and low incidence of relapse suggest that MRD negativity after CAR-T therapy followed by haplo-HSCT is an effective option for patients with R/R ALL. Several studies have reported that intermediate or high risk identified through risk stratification at diagnosis acts as a significant risk factor for OS and NRM in patients receiving allo-HSCT [21, 22]. Remarkably, multivariate analysis of our data showed that not only risk stratification at diagnosis but also several classic risk factors (such as adverse cytogenetics, total number of relapses, or high leukocyte counts) were no longer associated with poor OS or LFS, which may be related to patient selection and therapy decisions.

Notably, to the best of our knowledge, the present study is the first report of a combined CAR-T and
haplo-HSCT strategy against high tumor burden of R/R ALL. CAR-T therapy contributing to pre-transplant MRD negativity plays an important role in favorable clinical outcomes. Haplo-HSCT as consolidation also has a positive effect. In general, haplo-HSCT could have the following advantages for patients: (1) a theoretically high donor availability of almost $100 \%$, (2) a less timeconsuming process of finding a donor, and (3) a superior graft-versus-leukemia (GVL) effect [23, 24]. In our previous prospective study, we developed a protocol for $\mathrm{T}$ cell-replete haplo-HSCT with low-dose anti-Tlymphocyte globulin and showed that high-risk patients receiving haplo-HSCT experienced protection against relapse. Our protocol provided clinical evidence supporting haplo-identical donors as first-line alternative donors, especially for high-risk patients [25], in line with studies at other centers [26, 27]. Because all patients undergoing CAR- $\mathrm{T}$ treatment are relapsed/refractory with high-risk cytogenetics and/or molecular abnormalities, in the present study, we developed a novel strategy using CAR-T cells for re-induction followed by haploHSCT for consolidation in patients with R/R ALL. Theoretically, CD19-targeted CAR-T cells would eradicate all CD19-positive leukemia cells; however, we cannot exclude the possibility that certain CD19-negative leukemia sub-clones exist. Considering the results of this study, CAR-T cytotoxicity and GVL effect could be attenuated by conditioning therapy. The synergistic effects of CAR-T and conditioning therapy would potentially eradicate leukemia cells. Hay et al. reported that for patients who received CAR-T therapy to achieve MRD negativity before allo-HSCT, the 2-year LFS and OS were $61 \%$ and $72 \%$, respectively. The 2 -year cumulative incidence of relapse was $17 \%^{7}$, implying a prolonged LFS and OS. The present study showed similar results supporting a combined efficacy of CAR-T therapy and haplo-HSCT. Our results also support that haploidentical immune cells exert a potent GVL effect in such a combination modality.

Recently, studies in adult patients with Ph-negative ALL have established that the initial MRD response is a strong prognostic factor. The German Multicenter Study Group for Adult ALL analyzed the largest cohort of adult ALL data to assess MRD in Ph-negative patients and reported that molecular response was the only parameter with a significant prognostic effect [28]. Other studies confirmed a strong and independent prognostic effect of MRD after induction and early consolidation treatment [29-31]. In the present study, we clearly identified pre-transplant MRD negativity as an important independent predictor of high LFS and OS. Our data support a beneficial modality of haplo-HSCT following CAR-T treatment in patients with R/R ALL. 
In addition, we showed that the experience of relapses before CAR-T therapy was another adverse factor for high LFS. In patients with more relapse scenarios, leukemia cells exhibited stronger chemotherapy resistance, more genetic mutations, and immune escape [32, 33]. Moreover, consistent with previous studies in patients who received haplo-HSCT after chemotherapy $[34,35]$, our study found that age over 40 years was an independent risk factor associated with poor OS [36].

Safety issues are major concerns associated with combination therapy including immunotherapy and HSCT. In the present study, we observed no increased risk of treatment-related complications or immune toxicities. Moreover, no patient died of treatment-related complications. The 100-day cumulative incidence of grade IIIIV aGVHD was less than $10 \%$ in our study, similar to previously reported values $[8,17,37]$. Allo-HSCT after CAR-T treatment does not seem to increase the risk of therapy-associated complications.

Infection was another severe complication despite CAR-T treatment or allo-HSCT. Park et al. reported that 22 of 53 adult patients experienced 26 infections within the first 30 days after CAR-T infusion, and three patients died of an infection-related cause [38]. Infection is a primary or contributing cause of death in more than half of patients who die in the follow-up period after allo-HSCT [39]. Slade et al. reported that $62 \%$ and $6 \%$ of patients experienced at least one bacterial and one invasive fungal infection, respectively [39], whereas in the present study, the rates were $14.0 \%$ and $5.3 \%$, respectively. Moreover, CMV viremia was detected in the present study and in another study [40]. Our results show that despite the severe CRS and long-term duration of pancytopenia, the novel protocol of CAR-T treatment combined with haplo-HSCT did not increase the risk of infection.

The present study has several limitations, including the retrospective nature, lack of common prospective transplant protocols among the reporting transplant centers, and limited sample size, which may affect the reliability of the statistical analysis. The choice of covariates for the multivariate analysis was constrained by the small number of observed events.

\section{Conclusions}

The present study illustrates the safety and efficacy profiles of a novel combination therapy strategy against $R / R$ ALL by using the combination of CAR-T cells for reinduction followed by haplo-HSCT for consolidation. We confirmed that achieving pre-transplant MRD negativity after CAR-T treatment is a suitable basis for haplo-HSCT. Our results suggest that CAR-T therapy followed by haplo-HSCT could further improve LFS and OS without increasing risks of treatment-related toxicities in a previously heavily treated population. Further research in randomized case-controlled studies with longer follow-up periods is required.

\section{Supplementary information}

Supplementary information accompanies this paper at https://doi.org/10. 1186/s13045-020-00873-7.

Additional file 1: Supplementary Table S1. The case number enrolled by each clinical center. Table S2. CAR-T therapy associated toxicities. Table S3. Univariate analyses for factors impacting LFS in patients who received CAR-T therapy followed by haplo-HSCT. Table S4. Univariate analyses for factors impacting OS in patients who received CAR-T therapy followed by haplo-HSCT. Table S5. Univariate analyses for factors impacting cumulative incidence of relapse in patients who received CART therapy followed by haplo-HSCT. Table S6. Transplant-associated complications. Figure S1. Cumulative incidence of grade III-IV acute graft versus host disease (aGVHD), chronic graft versus host disease (cGVHD) requiring systemic steroid therapy, CMV viremia and EBV viremia in transplant group.

\section{Abbreviations}

aGVHD: Acute graft-versus-host-disease; Allo-HSCT: Allogeneic hematopoietic stem cell transplantation; CAR-T: Chimeric antigen receptor T cell; cGVHD: Chronic graft-versus-host-disease; Cl: Confidence intervals; CMV: Cytomegalovirus; CR: Complete remission; CRS: Cytokine release syndrome; EBV: Epstein-Barr virus; GVHD: Graft-versus-host-disease; GVL: Graft versus leukemia; Haplo-HSCT: Haploidentical hematopoietic stem cell transplantation; HR: Hazard ratio; HSCT: Hematopoietic stem cell transplantation; LFS: Leukemia-free survival; MRD: Minimal residual disease; NRM: Non-relapse mortality; OS: Overall survival; Ph: Philadelphia chromosome; R/R ALL: Relapsed/refractory acute lymphoblastic leukemia

\section{Acknowledgements}

We are indebted to colleagues from the Bone Marrow Transplantation Center, The First Affiliated Hospital, School of Medicine, Zhejiang University, Zhejiang Province Engineering Laboratory for Stem Cell and Immunity Therapy, Institute of Hematology, Zhejiang University, Department of Hematology, Beijing Tiantan Hospital, Department of Hematology, Tianjin First Central Hospital, Department of Hematology, Shanghai Tongji Hospital, Department of Hematology, Guangdong Second Provincial General Hospital, Department of Hematology, Changhai Hospital of Shanghai, Department of Hematology, Xiangya Third Hospital, Department of Hematology, Shanghai Children's Medical Center, Department of Hematology, Shanghai General Hospital, Department of Hematology, Peking University Third Hospital, Department of Hematology, Zhujiang Hospital of Southern Medical University, Department of Hematology, Xinhua Hospital of Shanghai, Innovative Cellular Therapeutics Co, Ltd, and Shanghai YaKe Biotechnology Ltd. We especially thank all the patients who participated in the study, without whom this study would never have been accomplished.

\section{Authors' contributions}

Houli Zhao, Jieping Wei, and Guoqing Wei were the principal investigators of the study. Mohamad Mohty, He Huang, and Yongxian Hu coordinated and oversaw the study. Yunxiong Wei, Ping Li, Jing Huang, and Tao Wang performed the material collection; Yi Luo, Jimin Shi, Mingfeng Zhao, Aibin Liang, Qing Zhang, Jianmin Yang, Xin Li, Jing Chen, Xianmin Song, Hongmei Jing, Yuhua Li, Siguo Hao, Wenjun Wu, Yamin Tan, Jian Yu, Yanmin Zhao, and Xiaoyu Lai participated in sample collection and/or treatment of patients; Lei Xiao, and Alex H. Chang helped in technical support and manufacture of CAR-T cells. Houli Zhao, Jieping Wei, Guoqing Wei, Elaine Tan Su Yin, Arnon Nagler, Mohamad Mohty, He Huang, and Yongxian Hu were the major contributors in writing the manuscript. All authors read and approved the final manuscript.

\section{Funding}

This work was supported by grants from the National Natural Science Foundation of China $(81230014,81470341,81520108002$, 81500157), the Key Project of Science and Technology Department of Zhejiang Province 
(2018C03016-2), and the Key Research and Development Program of Zhejiang Province (2019C03016)

\section{Availability of data and materials}

Bone Marrow Transplantation Center, The First Affiliated Hospital, School of Medicine, Zhejiang University will share the study protocol and individual, deidentified participant data that underlie the results reported in this article. The data will be available beginning 9 months and ending 36 months following article publication. The data will be shared with investigators whose proposed use of the data has been approved by Bone Marrow Transplantation Center, The First Affiliated Hospital, School of Medicine, Zhejiang University. The data may be used for individual participant data meta-analysis. Proposals may be submitted up to 36 months following article publication and should be directed to huanghe@zju.edu.cn.

\section{Ethics approval and consent to participate}

This study was performed in accordance with the Declaration of Helsinki and was approved by the ethics review committee of each institution. All participants provided written informed consent.

\section{Consent for publication}

Not applicable

\section{Competing interests}

Authors declare no conflicts of interest related to this work.

\section{Author details}

'Bone Marrow Transplantation Center, The First Affiliated Hospital, School of Medicine, Zhejiang University, No.79 Qingchun Road, Hangzhou, China. ${ }^{2}$ Zhejiang Province Engineering Laboratory for Stem Cell and Immunity Therapy, Hangzhou, China. Institute of Hematology, Zhejiang University, Hangzhou, China. ${ }^{4}$ Department of Hematology, Beijing Tiantan Hospital, Capital Medical University, Beijing, China. ${ }^{5}$ Department of Hematology, Tianjin First Central Hospital, Tianjin, China. ${ }^{6}$ Department of Hematology, Shanghai Tongji Hospital, Shanghai, China. 'Department of Hematology, Guangdong Second Provincial General Hospital, Guangzhou, China. ${ }^{8}$ Department of Hematology, Changhai Hospital of Shanghai, Shanghai, China. ${ }^{9}$ Department of Hematology, Xiangya Third Hospital, Changsha, China. ${ }^{10}$ Department of Hematology, Shanghai Children's Medical Center, Shanghai, China. ${ }^{11}$ Department of Hematology, Shanghai General Hospital, Shanghai, China. ${ }^{12}$ Department of Hematology, Peking University Third Hospital, Beijing, China. ${ }^{13}$ Department of Hematology, Zhujiang Hospital of Southern Medical University, Guangzhou, China. ${ }^{14}$ Department of Hematology, Xinhua Hospital of Shanghai, Shanghai, China. ${ }^{15}$ Institut Paoli-Calmettes, Marseille, France. ${ }^{16}$ Innovative Cellular Therapeutics Co, Ltd, Shanghai, China. ${ }^{17}$ Shanghai YaKe Biotechnology Ltd, Shanghai, China. ${ }^{18} \mathrm{Hematology}$ and Bone Marrow Transplantation Division, Chaim Sheba Medical Center, Tel-Hashomer, Israel.

${ }^{19}$ Sorbonne University, Saint-Antoine Hospital, INSERM UMRs 938, Paris, France.

Received: 14 February 2020 Accepted: 8 April 2020

Published online: 04 May 2020

\section{References}

1. Gökbuget N, Stanze D, Beck J, Diedrich H, Horst HA, Hüttmann A, et al. Outcome of relapsed adult lymphoblastic leukemia depends on response to salvage chemotherapy, prognostic factors, and performance of stem cell transplantation. Blood. 2012;120:2032-41.

2. Inagaki J, Fukano R, Noguchi M, Kurauchi K, Tanioka S, Okamura J. Hematopoietic stem cell transplantation following unsuccessful salvage treatment for relapsed acute lymphoblastic leukemia in children. Pediatr Blood Cancer. 2015;62:674-9.

3. Park JH, Rivière I, Gonen M, Wang X, Sénéchal B, Curran KJ, et al. Long-term follow-up of CD19 CAR therapy in acute lymphoblastic leukemia. N Engl J Med. 2018:378:449-59.

4. Pan J, Niu Q, Deng B, Liu S, Wu T, Gao Z, et al. CD22 CAR T-cell therapy in refractory or relapsed $B$ acute lymphoblastic leukemia. Leukemia. 2019:33: 2854-66.

5. Hu Y, Wu Z, Luo Y, Shi J, Yu J, Pu C, et al. Potent anti-leukemia activities of chimeric antigen receptor-modified T cells against CD19 in Chinese patients with relapsed/refractory acute lymphocytic leukemia. Clin Cancer Res. 2017; 23:3297-306.

6. Zhang LN, Song Y, Liu D. CD19 CAR-T cell therapy for relapsed/refractory acute lymphoblastic leukemia: factors affecting toxicities and long-term efficacies. J Hematol Oncol. 2018;11:41.

7. Hay KA, Gauthier J, Hirayama AV, Voutsinas JM, Wu Q, Li D, et al. Factors associated with durable EFS in adult B-cell ALL patients achieving MRDnegative CR after CD19 CAR T-cell therapy. Blood. 2019;133:1652-63.

8. Salvatore D, Labopin M, Ruggeri A, Battipaglia G, Ghavamzadeh A, Ciceri F, et al. Outcomes of hematopoietic stem cell transplantation from unmanipulated haploidentical versus matched sibling donor in patients with acute myeloid leukemia in first complete remission with intermediate or high-risk cytogenetics: a study from the acute leukemia working party of the European society for blood and marrow transplantation. Haematologica. 2018:103:1317-28

9. Hu Y, Sun J, Wu Z, Yu J, Cui Q, Pu C, Liang B, Luo Y, Shi J, Jin A, Xiao L, Huang $\mathrm{H}$. Predominant cerebral cytokine release syndrome in CD19directed chimeric antigen receptor-modified T cell therapy. J Hematol Oncol. 2016:9:70.

10. Code of manufacturing quality management for chimeric antigen receptor T cells (CAR-T cells) based-medicinal product. Chin Med Biotechnol, 2018;13: 386-394.

11. Jin M, Hu Y, Wu W, Luo Y, Tan Y, Yu J, et al. Decitabine plus CLAG chemotherapy as a bridge to haploidentical transplantation in the setting of acute myeloid leukemia relapse after HLA-matched sibling transplantation: a case report. BMC Cancer. 2019;19:242

12. Luo $Y$, Lai $X$, Tan $Y$, Shi J, Zhao $Y$, Han $X$, et al. Reduced-intensity allogeneic transplantation combined with imatinib mesylate for chronic myeloid leukemia in first chronic phase. Leukemia. 2009;23:1171-4.

13. Wang J, Luan Z, Jiang $H$, Fang J, Qin M, Lee V, et al. Allogeneic hematopoietic stem cell transplantation in thirty-four pediatric cases of mucopolysaccharidosis-a ten-year report from the China children transplant group. Biol Blood Marrow Transplant. 2016;22:2104-8.

14. Belkacemi Y, Labopin M, Giebel S, Loganadane G, Miszczyk L, Michallet M, et al; Acute Leukemia Working Party of the European Society for Blood and Marrow Transplantation (EBMT). Single-dose daily fractionation is not inferior to twice-a-day fractionated total-body irradiation before allogeneic stem cell transplantation for acute leukemia: a useful practice simplification resulting from the SARASIN study. Int J Radiat Oncol Biol Phys 2018;102:515-526.

15. Salter Al, Pont MJ, Riddell SR. Chimeric antigen receptor-modified T cells: CD19 and the road beyond. Blood. 2018;131:2621-9.

16. Tomuleasa C, Fuji S, Berce C, Onaciu A, Chira S, Petrushev B, et al. Chimeric antigen receptor T-cells for the treatment of B-cell acute lymphoblastic leukemia. Front Immunol. 2018;9:239. https://doi.org/10.3389/fimmu.

17. Zhao J, Song Y, Liu D. Clinical trials of dual-target CAR T cells, donor-derived CAR T cells, and universal CAR T cells for acute lymphoid leukemia. J Hematol Oncol. 2019;12:17.

18. Zhao XS, Liu YR, Xu LP, Wang Y, Zhang XH, Chen H, et al. Minimal residual disease status determined by multiparametric flow cytometry pretransplantation predicts the outcome of patients with ALL receiving unmanipulated haploidentical allografts. Am J Hematol. 2019;94:512-21.

19. Lovisa F, Zecca M, Rossi B, Campeggio M, Magrin E, Giarin et al. Pre- and posttransplant minimal residual disease predicts relapse occurrence in children with acute lymphoblastic leukaemia. Br J Haematol 2018;180: 680-693.

20. Pavlů J, Labopin M, Niittyvuopio R, Socié G, Yakoub-Agha I, Wu D, et al. Measurable residual disease at myeloablative allogeneic transplantation in adults with acute lymphoblastic leukemia: a retrospective registry study on 2780 patients from the acute leukemia working party of the EBMT. $J$ Hematol Oncol. 2019;12:108.

21. Saraceni F, Labopin M, Gorin NC, Blaise D, Tabrizi R, Volin L, et al. Matched and mismatched unrelated donor compared to autologous stem cell transplantation for acute myeloid leukemia in first complete remission: a retrospective, propensity score-weighted analysis from the ALWP of the EBMT. J Hematol Oncol. 2016;9:79.

22. Zhang M, Fu H, Lai X, Tan Y, Zheng W, Shi J, et al. Minimal residual disease at first achievement of complete remission predicts outcome in adult patients with Philadelphia chromosome-negative acute lymphoblastic leukemia. PLoS One. 2016;11:e0163599.

23. Hu YX, Cui Q, Liang B, Huang H. Relapsing hematologic malignancies after haploidentical hematopoietic stem cell transplantation. Biol Blood Marrow Transplant. 2011;17:1099-111. 
24. Xu L, Chen H, Chen J, Han M, Huang H, Lai Y, et al. The consensus on indications, conditioning regimen, and donor selection of allogeneic hematopoietic cell transplantation for hematological diseases in Chinarecommendations from the Chinese society of hematology. J Hematol Oncol. 2018;11:33.

25. Luo Y, Xiao H, Lai X, Shi J, Tan Y, He J, et al. T-cell-replete haploidentical HSCT with low-dose anti-T-lymphocyte globulin compared with matched sibling HSCT and unrelated HSCT. Blood. 2014;124:2735-43.

26. Brissot E, Labopin M, Ehninger G, Stelljes M, Brecht A, Ganser A, et al. Haploidentical versus unrelated allogeneic stem cell transplantation for relapsed/refractory acute myeloid leukemia: a report on 1578 patients from the acute leukemia working party of the EBMT. Haematologica. 2019;104:524-32.

27. Giannotti F, Labopin M, Shouval R, Sanz J, Arcese W, Angelucci E, et al. Haploidentical transplantation is associated with better overall survival when compared to single cord blood transplantation: an EBMT-Eurocord study of acute leukemia patients conditioned with thiotepa, busulfan, and fludarabine. J Hematol Oncol. 2018;11:110.

28. Gökbuget N, Kneba M, Raff T, Trautmann H, Bartram CR, Arnold R, et al. Adult patients with acute lymphoblastic leukemia and molecular failure display a poor prognosis and are candidates for stem cell transplantation and targeted therapies. Blood. 2012;120:1868-76.

29. Bassan R, Spinelli O, Oldani E, Intermesoli T, Tosi M, Peruta B, et al. Improved risk classification for risk-specific therapy based on the molecular study of minimal residual disease (MRD) in adult acute lymphoblastic leukemia (ALL). Blood. 2009;113:4153-62.

30. Ribera JM, Oriol A, Morgades M, Montesinos P, Sarrà J, González-Campos J, et al. Treatment of high-risk Philadelphia chromosome-negative acute lymphoblastic leukemia in adolescents and adults according to early cytologic response and minimal residual disease after consolidation assessed by flow cytometry: final results of the PETHEMA ALL-AR-03 trial. J Clin Oncol. 2014;32:1595-604.

31. Beldjord K, Chevret S, Asnafi V, Huguet F, Boulland ML, Leguay T, et al. Oncogenetics and minimal residual disease are independent outcome predictors in adult patients with acute lymphoblastic leukemia. Blood. 2014; 123:3739-49.

32. Paguirigan AL, Smith J, Meshinchi S, Carroll M, Maley C, Radich JP. Singlecell genotyping demonstrates complex clonal diversity in acute myeloid leukemia. Sci Transl Med. 2015;7:281re2.

33. Sood R, Hansen NF, Donovan FX, Carrington B, Bucci D, Maskeri B, et al. Somatic mutational landscape of AML with inv(16) or t(8;21) identifies patterns of clonal evolution in relapse leukemia. Leukemia. 2016;30:501-4.

34. Piemontese S, Boumendil A, Labopin M, Schmid C, Ciceri F, Arcese W, et al. Leukemia relapse following unmanipulated haploidentical transplantation: a risk factor analysis on behalf of the ALWP of the EBMT. J Hematol Oncol. 2019;12(1):68.

35. Bassan R, Hoelzer D. Modern therapy of acute lymphoblastic leukemia. J Clin Oncol. 2011;29:532-43.

36. Aoki J, Kanamori H, Tanaka M, Yamasaki S, Fukuda T, Ogawa H, et al. Impact of age on outcomes of allogeneic hematopoietic stem cell transplantation with reduced intensity conditioning in elderly patients with acute myeloid leukemia. Am J Hematol. 2016;91:302-7.

37. Wang Y, Wu DP, Liu QF, Xu LP, Liu KY, Zhang XH, et al. Low-dose post-transplant cyclophosphamide and anti-thymocyte globulin as an effective strategy for GVHD prevention in haploidentical patients. J Hematol Oncol. 2019;12:88.

38. Park JH, Romero FA, Taur Y, Sadelain M, Brentjens RJ, Hohl TM, et al. Cytokine release syndrome grade as a predictive marker for infections in patients with relapsed or refractory B-cell acute lymphoblastic leukemia treated with chimeric antigen receptor T cells. Clin Infect Dis. 2018;67:533-40

39. Slade M, Goldsmith S, Romee R, DiPersio JF, Dubberke ER, Westervelt P, et al. Epidemiology of infections following haploidentical peripheral blood hematopoietic cell transplantation. Transpl Infect Dis. 2017;19. https://doi. org/10.1111/tid.12629.

40. Avery RK, Silveira FP, Benedict K, Cleveland AA, Kauffman CA, Schuster MG et al. Cytomegalovirus infections in lung and hematopoietic cell transplant recipients in the organ transplant infection prevention and detection study: a multi-year, multicenter prospective cohort study. Transpl Infect Dis. 2018; 20:e12877.

\section{Publisher's Note}

Springer Nature remains neutral with regard to jurisdictional claims in published maps and institutional affiliations.

Ready to submit your research? Choose BMC and benefit from:

- fast, convenient online submission

- thorough peer review by experienced researchers in your field

- rapid publication on acceptance

- support for research data, including large and complex data types

- gold Open Access which fosters wider collaboration and increased citations

- maximum visibility for your research: over $100 \mathrm{M}$ website views per year

At BMC, research is always in progress.

Learn more biomedcentral.com/submissions 